\title{
The durability of prestressed concrete elements reinforced with fibres
}

\author{
V. COROBCEANU and R. GIUŞCĂ* \\ Faculty of Civil Engineering and Building Services, Technical University "Gheorghe Asachi” of Iasi \\ 67 Dimitrie Mangeron St., 700050 Iaşi, Romania
}

\begin{abstract}
The aim of the study is to show the improvements on durability of prestressed concrete elements by introducing in concrete mass a certain quantity of fibres. Random distributed fibres provide a significant increase in the tensile strength, having also favourable consequence on other mechanical and durability characteristics. The prestressed concrete elements are under a permanent stress state that is not optimal in all their sections and zones. One of the most unfavourable effects is the reduction of material durability, especially in aggressive environments, in which prestressed reinforcements are more sensitive to utilization than reinforced concrete components. The authors present the result brought up by the use of steel fibres in the improvement of the durability of concrete elements, in relation with the volumetric steel ratio, and the geometrical ratio of steel fibres, leading to a favourable effect on prestressing efficiency.
\end{abstract}

Key words: durability, prestressed concrete, fibres.

\section{Introduction}

The increase of performances of the main material which assures effectively the solving of the strength structures of the most diversified types of constructions has constituted, for a long time, a constant concern of the researches and practitioners. No matter of the form in which it is used (simple, slightly reinforced or prestressed) the concrete represents the most utilized construction material of this end of millennium, and the prognosis studies indicate it as principal favourite of the constructors in future, as well [1-11]. If in the last century, at the world level, the average amount of concrete consumed per capita of people was of about $0.75 \mathrm{~m}^{3}$, for the year 2012 this amount was $1.6 \mathrm{~m}^{3}$.

But, as it is well known, one of the essential disadvantages of the concrete is that referred to its low tensile strength, and to its brittle character of failure to its load, as a result of the development of the very little limit elongation. As a result, the utilization domains of that material are strictly limited to massive elements, which work mainly at compression [8].

The association of concrete with passive reinforcement solves partially this disadvantage. It is true that the utilization domains of the reinforced concrete increase spectacularly, but the behaviour in exploitation of the elements remains dependent on the above-mentioned parameter. For the rational utilization of the reinforcement, under service loads, the elements made by reinforced concrete work with tensioned cracked zones, which determines an important decrease of the stiffness and of the durability characteristics.

The utilization of the initial stresses has as a consequence an impressive increase of the material performances, and the behaviour in exploitation of the elements depends to a lesser extent on the value of the tensile strength of the used concrete [1].
Nevertheless, especially for the elements subjected to bending, the efficiency of the prestressing depends on those strength characteristics of the material.

At the same time, the durability of the elements in decisively influenced by the state of micro and macro - cracking of the concrete in transfer and in exploitation, as in the end zones highly loaded by efforts applied on reduced surfaces.

These are some of the reasons for which in the modern times of the constructions, in which these are exploited in most diversified mediums, it is necessary to use some concretes with a better tensile strength and a ductile behaviour [9].

One of the solutions which solves favourably that desideratum is given by the disperse reinforcement of the concrete, by introducing in that material mass of a quantity of discontinuous fibres of organic or inorganic provenience [11].

\section{The disperse reinforcement effects}

The idea of the disperse reinforcements of building materials, with the purpose of improving those physical-mechanical characteristics and those durability, has been known from the ancient times. Recent development has introduced the asbestos-cement, water and asbestos fibres, with a wide utilization in constructions and installations.

As for the last 30 years the amount of asbestos consumed has been in a continuous increasing, it is appreciated that world's reserves will be finally depleted.

As a result, the sixth decade of the last century began, in the western countries with organized researches and tests having in view the utilization of some new types of fibres (steel, glass, alkali-resistant, carbon or plastics).

The main physical - mechanical properties of the fibres, which can be used in disperse reinforcing of the concrete are presented in Table 1.

\footnotetext{
*e-mail: rgiusca@yahoo.com
} 
Table 1

Physical - mechanical characteristics of fibres

\begin{tabular}{cccccc}
\hline \hline Fibres type & Diameter d, $\mu$ & $\begin{array}{c}\text { Density } \\
\mathrm{Pa} \mathrm{kg} / \mathrm{m}^{3}\end{array}$ & $\begin{array}{c}\text { Tensile strength } \\
\mathrm{R}_{t}, \mathrm{GPa}\end{array}$ & Elasticity modulus GPa & $\begin{array}{c}\text { Breaking elongation } \\
\delta_{r} \%\end{array}$ \\
\hline Asbestos & $0.02-20$ & $\sim 3200$ & $0.5-3.0$ & $80-150$ & $0.5-2.0$ \\
\hline Cardboard & $8-9$ & 1900 & $1.8-2.6$ & $200-300$ & $0.5-1.0$ \\
\hline Steel & $5-800$ & 7850 & $1.0-3.0$ & 210 & $3-4$ \\
\hline Glass & $9-15$ & $\sim 2500$ & $1.0-4.0$ & $70-80$ & $0.95-1.37$ \\
\hline Alumina & $500-770$ & $\sim 3900$ & 0.65 & 245 & $0.13-0.39$ \\
\hline Polyethylene & $20-200$ & 950 & 0.7 & $0.14-0.42$ & $0.40-1.60$ \\
\hline Polypropylene & $20-200$ & 900 & $0.5-0.8$ & $3.5-5.0$ & 0.17 \\
\hline Polyester & $20-200$ & 950 & $0.7-0.9$ & 8.4 & -0.74 \\
\hline
\end{tabular}

In the last column of this table there is given the quality coefficient (tensile strength) apparent density, from which there results the fact that, from this point of view, the most efficient are glass and carbon fibres.

At the same time mention must be made of the fact that a very important technical characteristic is the surface nature. In the case of asbestos carbon and glass fibres, superior adherence between fibre and matrix results in a high sliding resistance.

The first obvious effect, of the concrete's fibrering is increase of the tensile strength. Function of the type of fibre used, of the adherence to matrix, reinforcing percentage and geometric ratio $(1 / \mathrm{d})$ this parameter may be increased by two or five compared to that of the non-reinforced matrix, with visible consequence for the behaviour until breaking of the members thus make - up.

Besides, concrete with disperse reinforcing has ductile breaking, very good impact behaviour, dynamic loads and figure and a great wear strength [7].

Modifying the macro and macro - cracking character of the matrix, the durability characteristics of that result much improved.

The utilisation of these concretes to realize prestressed concrete members determines a superior protection of the active reinforcement than in case if classic concrete.

It is not negligible the fact that fibre concretes to increase the efficiency of the prestressing and determine the increasing of the capable bending moment, and also an advantageous stress state both at transfer and in service.

\section{Steel - fibres matrix solution}

Despite of a more diminished quality coefficient, our experiences were orientated to the fibres of steel that is easy to be used in Romania's conditions. Its adherence to cement matrix (concrete) was thoroughly studied.

In order for the disperse reinforcement to achieve the desirable effect, the length of fibres, in the conditions of realizing an acceptable minimum adherence, must have at least a minimum value.
Let it be a segment from a matrix which has a single fibre, presented in Fig. 1, orientated after tensioned efforts directions which stresses matrix, " $\sigma_{m}$ ".

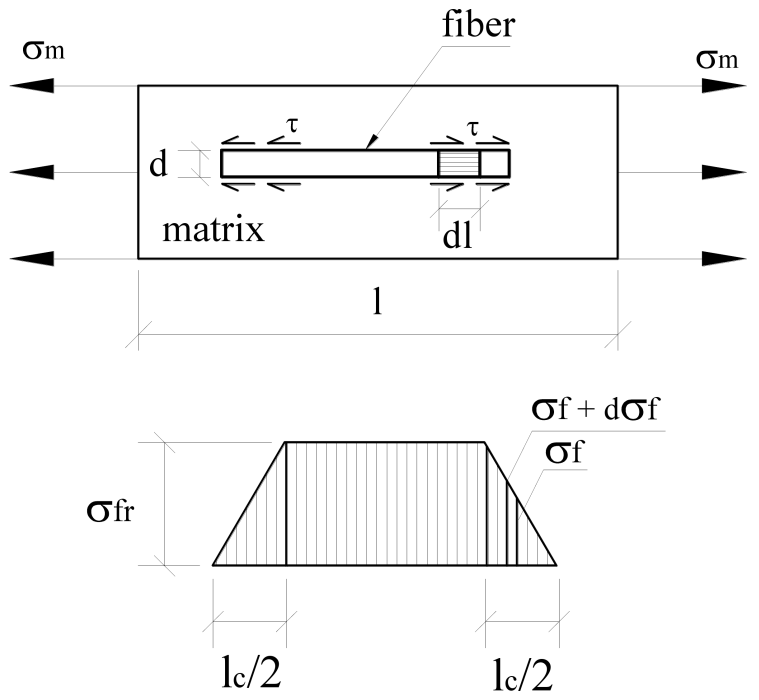

Fig. 1. Stress distribution in fibre

The transmission of the efforts from matrix to fibre is made by adherence tangential stresses " $\tau$ ", so that the stress from fibres increases zero to the failure value " $\sigma_{f r}$ ".

If we consider differential elements of fibre of length " $d l$ ", it may be written:

$$
\frac{\pi \cdot d^{2}}{4} d \cdot \sigma_{f}=\pi \cdot d \cdot \tau_{m} \cdot d l
$$

By integrating this equation in given condition from Fig. 1, it results:

$$
l_{c}=\frac{d \cdot \sigma_{f r}}{2 \cdot \tau_{m}}
$$

where $1_{c}$ - represents the fibre critic length so as its stress should touch the failure one.

Normal stress from fibre " $\sigma_{f}$ " and that of adherence " $\tau$ " vary, for different lengths of fibres as shown in Fig. 2, while adherence stress distribution when a crack occurs in matrix is illustrated in Fig. 3. 


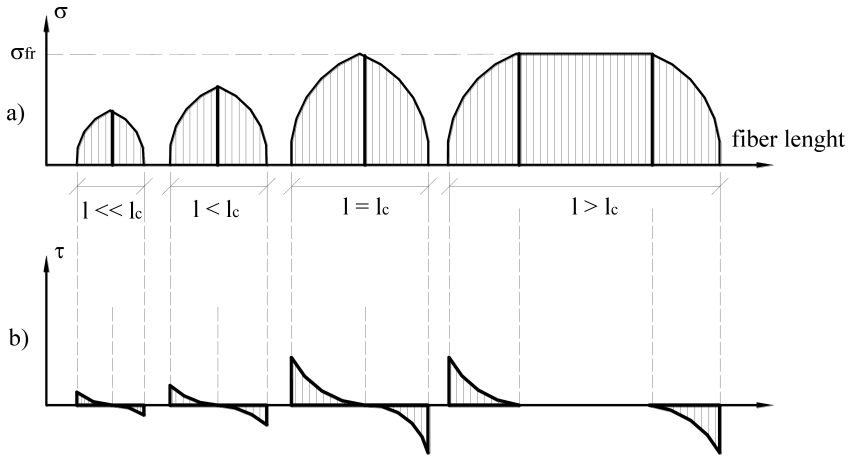

Fig. 2. Schematic variation of stresses a) In fibre b) Of adherence
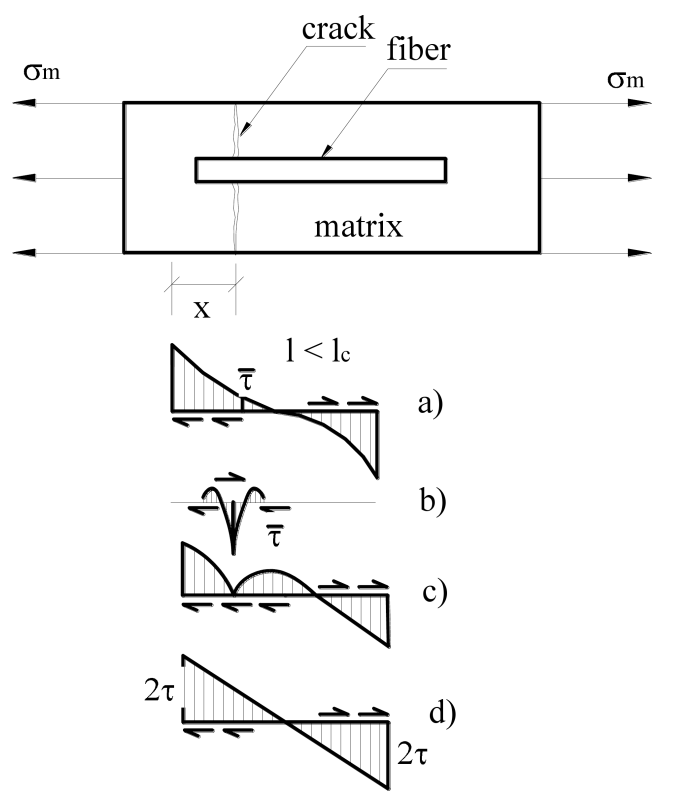

Fig. 3. Distribution of adherence stresses before and after occurrence of crack in matrix: a) Before b) After cracking c) Combined effect of two diagrams d) Idealised diagram

The fibres minimal length, in order to have an effect on the elements self-carrying capacity may be calculated with the aid of the following relation:

$$
l=2 \cdot l_{c}
$$

This relation can be estimated on the basis of the following reasoning. At the occurrence of a crack in matrix, the fibre crosses it, and the smaller embedded end is the most important for post-cracking behaviour. It is assumed that the most likely value of fibre short length is $1 / 4$. If this is less or equal to $l_{c} / 2$, it produces the fibre pullout from a matrix, without " $\sigma_{f}$ " touching " $\sigma_{f r}$ ".

On the contrary, if $l_{f}<2 l_{c}$, after cracking, the matrix accepts a new increase of stresses.

Then the minimum geometrical ratio can be also calculated so no pulling-out of fibres should occur at the appearance of the first crack:

$$
\frac{l}{d}=\frac{R_{t}}{\tau} \cdot \frac{E_{f}}{E_{b}}
$$

where $R_{t}$ - is stress from fibre which corresponds to the appearance of the first crack, $E_{f}, E_{b}$ - elasticity modulus of fibre, and of matrix respectively.
As we can observe, this ratio is influenced by the fibre type, its surface and physical-mechanical characteristics of matrix, a very important conclusion that has determined the way in which our works were done.

\section{Materials used - results obtained in increasing mechanical characteristics}

For matrix natural aggregates with dimensions $0 \ldots 7.1 \mathrm{~mm}$ were used, divided in two sorts: $0 \ldots 3 \mathrm{~mm}$ (minimum $50 \%$ from the quantity of aggregate necessary for ensuring a better workability) and 3...7.1 mm. The cement used was Portland II/A-S $32.5 \mathrm{R}$ type.

In these conditions, the mixture proportions for one cubic meter of concrete were:

Aggregate 0...3 mm: $670 \mathrm{~kg}$;

Aggregate 3...7.1 mm: $620 \mathrm{~kg}$;

Cement II/A-S $32.5 \mathrm{R}: 735 \mathrm{~kg}$;

Water $(\mathrm{W} / \mathrm{C}=0.35): 257 \mathrm{l}$;

Super - plasticizer: 7.351 .

Steel fibres were obtained by cutting laminated wires, having diameter $0.4 \mathrm{~mm}$, with breaking limit $\sigma_{r}=880 \mathrm{MPa}$ and breaking elongation $\varepsilon=22 \%$.

The provisions given by a recommend that the diameter of fibres is to be between 0.2 and $0.5 \mathrm{~mm}$, and geometric ratio $1 / \mathrm{d}$ around 100 to avoid segregation and their agglomeration, and to obtain a uniform mixture with homogenous dispersion. Preliminary tests showed that for the wire with $0.4 \mathrm{~mm}$ diameter, $40 \mathrm{~mm}$ length of fibres determined by the ratio $1 / \mathrm{d}=$ 100 still results in occurrence of such phenomena.

For this reason we adopted $25 \mathrm{~mm}$ length $(1 / \mathrm{d}=62.5)$ and we used two types of longitudinal profile of fibres: straight and finely waved, the last being chosen for ensuring a superior adherence to matrix.

Volumetric steel ratios "P" ranged between 1 and 4\%, every 0.5 . Concrete pouring was realised on the vibrating table with limited vibration time, to avoid the concentration of fibres in certain zones of test - samples.

Their free surfaces were finished to remove the fibres, which go out from the fresh concrete mass. The storage conditions and test time (28 days) were the same for all types of specimens [2].

For each concrete mixture, witness and with fibres realised with the above-mentioned steel ratio, the following types of specimens were made:

- Cubes of $141 \mathrm{~mm}$ sides, for determining the compressive strength and frost deftness;

- Cylinders of $100 \mathrm{~mm}$ diameter and length of $251 \mathrm{~mm}$ for determining split-strength and the elasticity modulus;

- Prisms of $100 \times 100 \times 550 \mathrm{~mm}$ size, for determining the strength to bending tension and the deflection for the same load, and also for determining some characteristics of durability;

- Plates of $200 \times 200 \times 10 \mathrm{~mm}$ size, for determining the shock resistance.

- The values of mechanical strengths and those of bending deformability are given in Table 2 . 
Table 2

Mechanical characteristics of steel fibre reinforced concrete

\begin{tabular}{|c|c|c|c|c|c|}
\hline \multirow{3}{*}{ Fibre type } & \multirow{3}{*}{ Steel ratio vol. $\mathrm{p} \%$} & \multicolumn{4}{|c|}{ Medium values of mechanical characteristics } \\
\hline & & \multirow{2}{*}{$\begin{array}{c}\text { Compression } \\
\mathrm{R}_{c} \mathrm{MPa}\end{array}$} & \multicolumn{2}{|c|}{ Tension } & \multirow{2}{*}{$\begin{array}{c}\text { Deflection } \\
\mathrm{mm}\end{array}$} \\
\hline & & & Split Rtd & Bending Rti & \\
\hline Witness & - & 51.2 & 3.9 & 6.2 & 0.13 \\
\hline \multirow{7}{*}{$\begin{array}{c}\text { Straight fibres } \\
d=0.4 \mathrm{~mm} \\
l / d=62.5\end{array}$} & 1.0 & 51.8 & 4.4 & 8.36 & 0.29 \\
\hline & 1.5 & 53.2 & 4.9 & 9.96 & 0.38 \\
\hline & 2.0 & 55.8 & 6.7 & 11.90 & 0.56 \\
\hline & 2.5 & 57.6 & 7.8 & 13.80 & 0.64 \\
\hline & 3.0 & 58.8 & 8.2 & 15.64 & 0.71 \\
\hline & 3.5 & 60.2 & 8.9 & 17.28 & 0.79 \\
\hline & 4.0 & 62.8 & 9.6 & 18.66 & 0.85 \\
\hline \multirow{7}{*}{$\begin{array}{l}\text { Waved fibres } \\
d=0.4 \mathrm{~mm} \\
l / d=62.5\end{array}$} & 1.0 & 52.0 & 5.0 & 9.2 & 0.377 \\
\hline & 1.5 & 53.1 & 5.5 & 11.5 & 0.502 \\
\hline & 2.0 & 54.3 & 7.4 & 15.2 & 0.750 \\
\hline & 2.5 & 57.6 & 8.6 & 17.5 & 0.877 \\
\hline & 3.0 & 58.9 & 9.2 & 19.8 & 0.980 \\
\hline & 3.5 & 61.2 & 9.9 & 21.8 & 1.051 \\
\hline & 4.0 & 62.6 & 10.3 & 27.1 & 1.122 \\
\hline
\end{tabular}

The presence of fibres in matrix affected especially the tensile strength. Its increments are very important even for average steel ratios $p=1.5 \ldots 2 \%$ as it results from Fig. 4 .

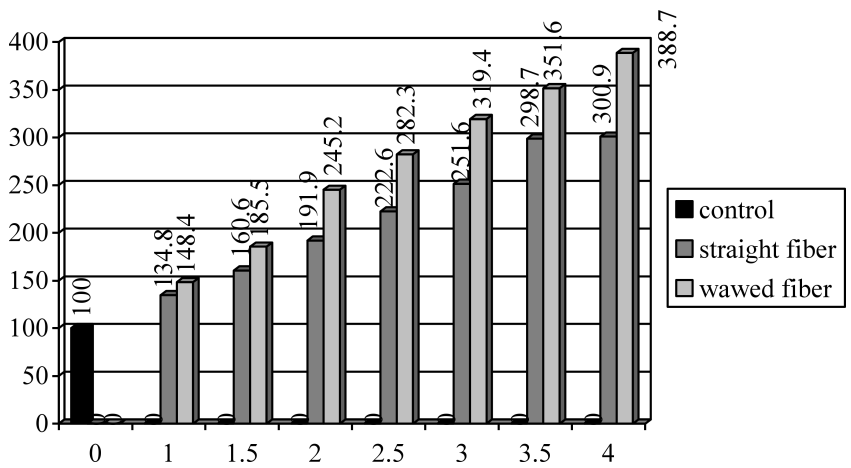

Fig. 4. Increase of tension by bending strength function of fibre profile and of steel ratio

The modification of $\mathrm{R}_{t} / \mathrm{R}_{c}$ ratio in the case of non - reinforced matrix to 0.21 in the case of matrix reinforced with straight fibres $(p=2 \%)$ and to 0.28 for the same ratio in the case of waved fibres, has very important practice consequences.

At the same time, as it resulted from the microscopic analysis of some samples subjected to variable stresses the micro reinforcing of concrete essentially modifies the value of stresses at which micro - cracking occurs, as well as its character.

Micro - cracks occur at higher stages of loading, related to failure strength, they have a homogeneous distribution in matrix mass and they develop in smaller openings compared to non - reinforced witness.

This aspect has immediate consequences for all durability characteristics of micro - reinforced concrete, especially for that used in realising prestressed members.
Analysing some of these characteristics in members subjected to ever increasing stresses produced by prestressed force, left us without doubts that we reached these conclusions.

\section{Utilisation of concretes with fibres to prestressed concrete members}

It is well known that in members with initial stresses, the efficiency of prestressing (cracking moment and breaking moment, consumption of active and passive steel, stress state in service and at transfer in extreme fibres, etc.) is also determined by the value of the prestress force eccentricity, by the level of internal forces couple, respectively. In its turn, the eccentricity is determined, among others, by the level of tensile strength necessary for the superior and inferior concrete fibres, at transfer and in exploitation [1].

For members with constant moment of inertia and with straight active steel reinforcing, the value of the tensile strength for the used concrete becomes decisive in what concerns the position on the section of the latter, with all favourable or unfavourable consequences that evolve from this positioning. Its results very clearly that for ensuring the arrangement of active steel as close to element fibres as possible, for obtaining the well-known advantages in service, the tensile strength in the opposite fibre must be high as possible for getting over, in good conditions, the stress state created at transfer

Furthermore, in zones of simple or articulated support the stress state produced at transfer practically remains unmodified on their entire service live, stresses being unfavourable for the material most of the time (tensions which exceed " $R_{t}$ ", which determine the cracking of top fibres followed by important reductions in rigidity and durability characteristics).

In order to totally avoid these disadvantages, the practice requirements ask for the using concretes with higher tensile 
strengths, and from this point of view, the concretes with fibres represent a very reasonable solution [6].

From the very ample programme of researches that aimed at explaining many more aspects, we have chosen some examples that support the subject of this paper. For the investigations on members we decided for some $T$ cross - section beams, as shown in Fig. 5, with active steel 6x3Ø2 SBP I, having $\sigma_{r}=2050 \mathrm{MPa}$, stretched before concrete pouring with $\sigma_{p k}=1640 \mathrm{MPa}$. For realising the beams, no passive reinforcements were used (longitudinal, transversal or local), for a clear watching of the effect of fibres.

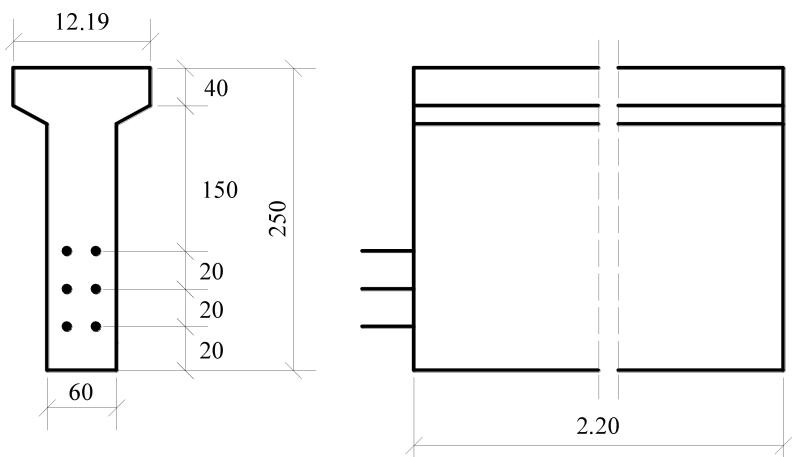

Fig. 5. Cross-section and longitudinal profile of beams

For analysing the types of samples we chose:

- Witness beams without fibres;

- Beams of concrete with fibres $p=1.5 \%$ (waved fibres);

- Beams of concrete with fibres $p=3 \%$ (waved fibres).

In what concerns the behaviour at transfer and then at failure of these beams the following observation significant for analysing-their durability are shortly made evident:

a. The witness beam presented at transfer obvious cracks in top fibres, with openings under $0.12 \mathrm{~mm}$ in zones afferent to supports and of about $0.10 \ldots 0.11 \mathrm{~mm}$ in central portions. At the same time, due to the absence of local reinforcement, longitudinal cracks, parallel to the direction of maximum compressive stresses at transfer, were observed. Under loads that represent $75 \%$ of that of failure, that are equivalent to service loads, the witness beam presented normal cracks in central inferior zone, with openings between 0.08 and $0.11 \mathrm{~mm}$. The breaking was brittle, showing very clearly a non-ductile behaviour.

b. The prestressed beam with $p=1.5 \%$ had a better behaviour than the witness, even at transfer and in service. The cracking of top parts from supports had a random character, the cracks opening being limited to $0.05 \mathrm{~mm}$, and in exploitation normal cracks did not exceed $0.04 \mathrm{~mm}$. Inclined cracks did not occur. The brittle character at failure was visibly attenuated, the deflection at failure increased 2.35 times, and breaking moment was 1.49 times bigger than in the witness (Fig. 6b).

c. The prestressed beam with $p=3 \%$ did not present cracks at transfer, and the slippage of active steel in end zones of the member was minimum.

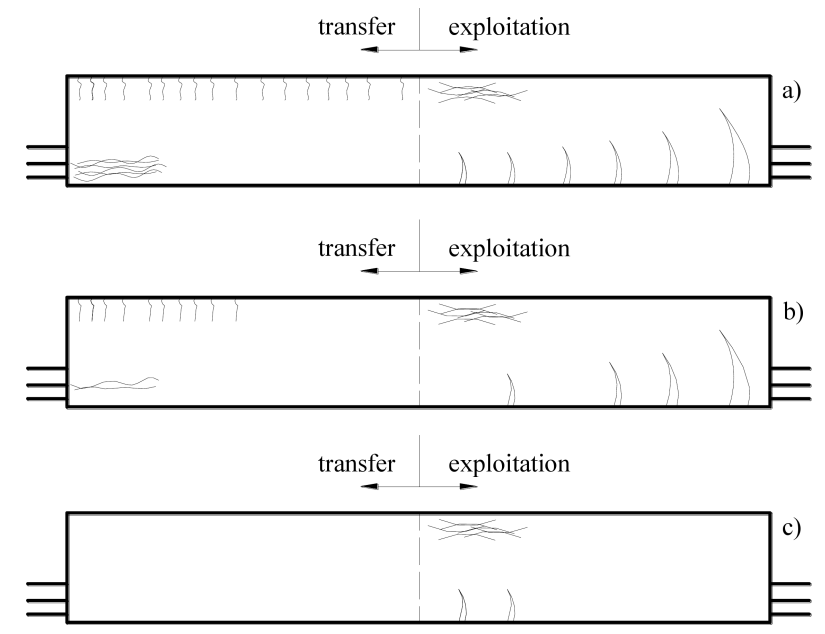

Fig. 6. Cracks survey for tested beams: a) Control b) $p=1.5 \%$ c) $p$ $=3 \%$

In exploitation the beam worked permanently under uncracked regime, and at failure, the deflection was 4.17 times bigger than that of the witness, and capable moment increased 3.48 times compared to the same witness (Fig. 6c).

\section{Durability of prestressed members with fibres}

Because the research is still unfolding, the beams with different ratios of fibres, subjected to more type of chemical aggressive medium, in the following will be presented only the results obtained up to now [3].

Characteristic for the industrial buildings realised in Romania there is an advanced state of corrosion in the structural reinforced concrete members, but especially in that of prestressed concrete, which made necessary ample works of consolidation. From among aggressive environments the most penetrating are those containing: sulphate, nitrites, chlorine, fat acids and the much mooted oil corrosion.

In the first stage we chose the study of chlorine corrosion on prestressed members, the analysis being made at the transfer and also under service loads. The corrosive environment solution was applied by pellicles establishing a correlation between its chlorine concentration and that found in industrial environment [4].

The studied samples were grouped by threes in closed precincts, also adding to each group an identical element as composition, but without prestressing stresses or service loads. In the same medium samples of cubic and prismatic shape were introduced.

There were analysed, at different periods, the following characteristics:

- Penetration level of chlorine ion in all elements and samples (results are given in Table 3);

- Decreasing of compressive strength for control samples at different periods, in comparison with witness samples, kept in standard conditions as shown in Table 3;

- Carrying capacity of members exposed to corrosive medium at final period, in comparison with that of members kept in normal conditions of serviceability; 
Table 3

Hardened concrete properties

\begin{tabular}{|c|c|c|c|c|c|c|c|c|c|}
\hline \multirow{3}{*}{ Mixture series } & \multirow{3}{*}{$\begin{array}{l}\text { Fibre reinforcing } \\
\text { percentage } \mathrm{p} \%\end{array}$} & \multirow{3}{*}{ Keeping environment } & \multirow{2}{*}{\multicolumn{3}{|c|}{$\begin{array}{c}\text { Compressive strength } \\
\mathrm{MPa}\end{array}$}} & \multirow{2}{*}{\multicolumn{3}{|c|}{$\begin{array}{c}\text { Flexure strength } \\
\mathrm{MPa} \\
\end{array}$}} & \multirow{3}{*}{$\begin{array}{c}\text { Chloride ion } \\
\text { penetration } 28 \mathrm{~d}\end{array}$} \\
\hline & & & & & & & & & \\
\hline & & & $14-d$ & $28-d$ & $42-d$ & 14-d & $28-d$ & $42-d$ & \\
\hline 1 & 0 & standard & 37.3 & 51.2 & 53.7 & 4.2 & 6.2 & 6.6 & - \\
\hline 2 & 1.5 & standard & 38.8 & 53.1 & 55.6 & 7.7 & 11.5 & 12.3 & - \\
\hline 3 & 3 & standard & 43.0 & 58.3 & 61.7 & 13.2 & 19.8 & 21.1 & - \\
\hline 4 & 0 & aggressive & 34.3 & 41.9 & 43.0 & 3.8 & 5.1 & 5.3 & 1986 \\
\hline 5 & 1.5 & aggressive & 36.5 & 47.3 & 48.4 & 7.2 & 10.2 & 10.7 & 1407 \\
\hline 6 & 3 & aggressive & 42.1 & 56.5 & 57.9 & 12.9 & 19.0 & 19.8 & 888 \\
\hline
\end{tabular}

- Structure and strength of the most unfavourably loaded concrete zone, analysed by non-destructive methods;

- Study of resistance and deformability characteristics of active reinforcements, made on samples extracted from members subjected to simulated corrosion process.

\section{Conclusions}

- Utilisation of steel fibres for disperses reinforcing concrete, for economic steel ratios, results firstly, in significant increasing of concrete tensile strength and the ductile behaviour till breaking [9];

- Micro - cracking character is essentially changed, i.e. the phenomenon occurs for loading stages 17 ...35\% higher; at the same time, the distribution of micro-cracks is uniform, with openings and lengths much lower in comparison with non-reinforced concrete [10];

- The above observation enables us to assume that the durability of such members subjected to different stress states is sensibly improved $[6,10]$;

- Utilisation of concrete with fibres for realising members with initial stress, beside the effect of the increasing prestressing efficiency, results in the increasing of their resistance to chemical aggressive environments;

- Mechanical strengths results of samples kept in aggressive medium show a diminishing of compressive and tensile strength function of the period of keeping, in comparison with the strength of those kept in standard conditions;

- At the same time the presence of fibres diminishes these losses in strength as the steel ratio increases;

- Analysis of samples extracted from tested specimens shows that steel fibres are affected by corrosive agent only in superficial zones;

- Penetration of chlorine ion sensibly decreases from witness to reinforced specimens with $p=1.5 \%$ and in a greater measure to those with $p=3 \%$;

- Favourable effect of fibre in concrete is felt to a greater extent in members with initial stresses, in zones most loaded, even at transfer and in service. Active and passive reinforcements are more protected in comparison with those of members without fibres, and their tendency of brittleness disappears almost completely;

- Breaking moment for prestressed members with fibres, exploited in aggressive environment, reduces to a smaller extent than for similar members realised of ordinary concrete;

- Results obtained after this stage of researches offer optimistic premises for their continuing and diversifying.

\section{REFERENCES}

[1] N. Rajagopalan, Prestressed Concrete, Alpha Science, Oxford, 2003.

[2] Y. F. Chen and E. M. Lui, Handbook of Structural Engineering, CRC Press, New York, 2005.

[3] M. Bărbuţă and D. Lepădatu, "Mechanical characteristics investigation of polymer concrete using mixture design of experiments and response surface method", J. Applied Sciences 8 (12), 2242-2249 (2008).

[4] M. Harja, M. Bărbuţă, L. Rusu, and N. Apostolescu, "Utilization of ash from thermal power plants in industry and environmental protection. I. ash characterization", Environmental Eng. and Management J. 7 (3), 289-293 (2008).

[5] A.M. Neville and D.J. Hannant, Fibre Reinforced Cement and Concrete, International Union of Testing and Research Laboratories for Materials and Structures", Int. Construction Press, University of Michigan, Michigan, 2007.

[6] R. Holland, Reinforced Concrete, Thomas Telford, London, 1997.

[7] L. Bejan, A. Sirbu, and N. Taranu, "Component properties influence upon the elasticity modules of the cloth - reinforced polymeric composites", Materiale Plastice J. 44 (1), 22-25 (2007).

[8] P. Mihai, I. Hîrhui, and B. Rosca, "Numerical analysis of bonding between concrete and reinforcement using the finite element method", J. Applied Sciences 10 (9), 738-744 (2010).

[9] G. Oprişan, N. Ţăranu, V. Munteanu, M. Budescu, C.I. Cozmanciuc, and R. Oltean, "Improvement of concrete strength through confining with composite membranes", Romanian J. Materials 4, 302-315 (2011).

[10] V. Corobceanu and R. Giuşcă, "The use of the fibreglass at the reinforced concrete elements", Buletinul Institutului Politehnic Iaşi L (LIV) 5, 81-86 (2004).

[11] M. Vasilache, M. Pruteanu, and C. Avram, "Use of waste materials for thermal insulation in buildings", Environmental Eng. and Management J. 9, 1275-1280 (2011). 